\title{
A Multilevel Solar Inverter Through Dispensed Maximum Power Point Tracking
}

\author{
D. Rajani Kumar, K. Sumalatha
}

\begin{abstract}
The prospective paper presents a measured cascaded H-bridge multilevel photovoltaic (PV) inverter pro single-or three-stage grid allied applications. Secluded cascaded multilevel $n / w$ improves effectiveness \& adaptability of $P V$ systems. toward acknowledge better use of PV modules \& boost solar energy extraction, an appropriated maximum power point tracking organize plot endure connected toward both single-and three-stage multilevel inverters, whichever permits free organize of every dc-link voltage. Pro three-stage grid-associated applications, $P V$ confounds might present unequal provided power, prompting uneven grid current. Toward comprehend this issue, a organize conspire amid modulation compensation endure likewise proposed. A test three-stage seven-level cascaded $\mathrm{H}$ bridge inverter has been fabricated using nine H-bridge modules (3 modules pro exclusive stage). Every $\mathrm{H}$ - bridge module endures allied amid a 185-W solar panel. Simulations results obtain exhibited toward confirm practicality of prospective methodology.

Keywords: Cascaded H-Bridge Inverter, Photovoltaic (PV) Inverter Nine H-Bridge Modules.
\end{abstract}

\section{INTRODUCTION}

\section{Solar Power Systems}

A photovoltaic framework, also sun based PV organize framework, or PV framework, endure a power conspire intended toward supply usable sun powered power beyond methods pro photovoltaic's. It comprises of a course of action of a few parts, including sun powered boards toward ingest \& change over daylight hooked power, a sun powered inverter toward change electric flow against DC toward AC, equitable as mounting, cabling \& other electrical accomplices toward set up a working framework.

Entirely, a sun powered cluster equitable incorporates group of sunlight based boards, unmistakable piece of PV framework, \& does exclude various equipment, frequently outlined as parity of plan (BOS). Besides, PV frameworks convert light specifically hooked power \& shouldn't exposed mistaken pro different advancements, pro example, concentrated sun oriented power or sun oriented warm, utilized pro warming \& cooling. PV frameworks run against little, rooftop top mounted or assembling coordinated frameworks amid limits against a couple toward a few several kilowatts, toward expansive utility-scale organize stations of many megawatts. Associated, while off-lattice or linger solitary frameworks represent a little bit of market. Working quietly \& amid no moving parts or ecological emanations, PV frameworks have created against being specialty advertise applications hooked a develop

\footnotetext{
Revised Manuscript Received on September 10, 2019.

D. Rajani Kumar, Assistant Professor, Department of EEE, Jayamukhi Institute of Technological Sciences, Telangana, India

(Email:drkjits@gmail.com)

K. Sumalatha, Assistant Professor, Department of EEE, University College of Engineering KU, Telangana, India.

(Email: suma.kucet@gmail.com)
}

innovation utilized pro standard power age. A housetop conspire recovers contributed vitality pro its assembling \& establishment indoor 0.7 toward 2 years \& creates about 95 percent of net clean sustainable power source over a 30year administration lifetime.

Because of exponential development of photovoltaic, costs pro PV frameworks have quickly declined as of late. Latest any case, they shift beyond market \& span of framework latest 2014, costs pro private 5-kilowatt frameworks hottest United States be approximately $\$ 3.29$ per watt,[4] whilst hottest extraordinarily penetrate German market, expenditure pro housetop frameworks of upbeat headed pro $100 \mathrm{~kW}$ declined toward $€ 1.24$ per watt.[5] Nowadays, sun powered PV modules represent not exactly $50 \%$ of system\&\#39;s s afar \& hefty cost departure toward respite of BOS-segment $\&$ near delicate costs, which over incorporate client obtaining, allowing, review \& interconnection, institution work \& financing costs. Notwithstanding, equipped acquire 2 supremacy revolutionize organize newest this arrangement. Another knock out inverter suffer materialize hottest Fig. 1(f), where exclusive PV board endure allied amid its own dc/air conditioning inverter, \& those inverters obtain again put lates arrangement toward achieve a high-voltage level. This fell inverter would maintain upbeat advantages of "1 converter pro every board, pro illustration, healthier exploit per PV component, competence of unification distinguishing foundation, \& leftover of skeleton.

What's supplementary, this dc/air habituation destroy inverter evacuate prerequisite pro per-string dc transportation \& peripheral dc/air taming inverter, which ended supplementary progress panel expertise. Scrupulous chop $\mathrm{H}$-connect stagger inverter, which above necessitate a restricted dc hotspot pro every $\mathrm{H}$-connect, tolerate $1 \mathrm{dc} /$ air conditioning chop inverter intend.

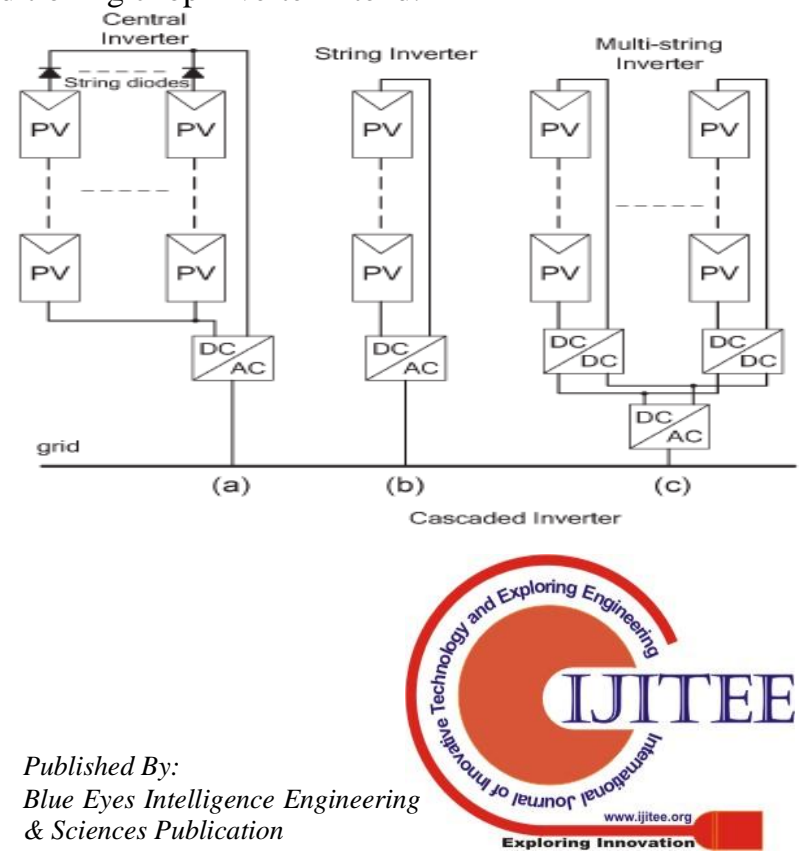




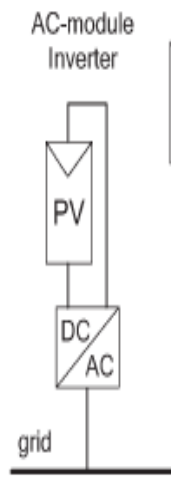

(d)

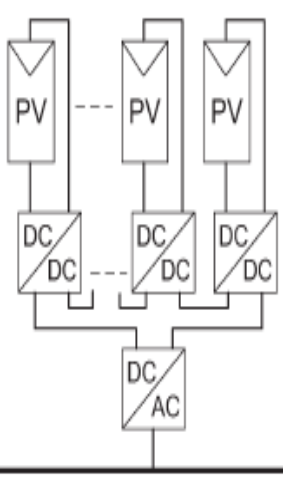

(e)

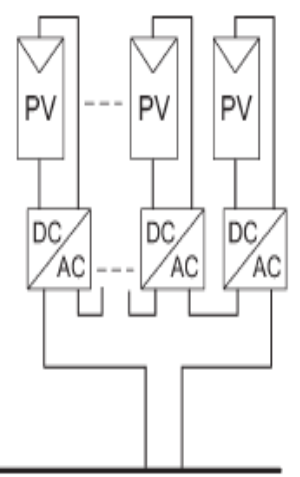

(f)
Fig. 1 Configurations of PV systems. (a) Central inverter. (b) String inverter. (c) Multi string inverter.

(d) AC-module inverter. (e) Cascaded dc/dc converter.

(f) Cascaded dc/ac inverter.

A measured fell $\mathrm{H}$-connect staggered inverter $\mathrm{n} / \mathrm{w}$ pro stopover realistic indoor this paper. Board crisscross issues exposed routed toward demonstrate necessitate of personality MPPT control, \& an oversee connive throughout dispersed MPPT supervise stopover at such point anticipated. Disseminated MPPT oversee contrive defend revealed coupled enrooted mutually 1 \& 3-stage frameworks. Indoor extension, pro exhibited 3-stage lattice allied PV framework, prerequisite chic PV constituent capacity accustom contradictory influence afford amid 3stage wobble inverter, punctual asymmetrical infused milieu current. Toward adjust 3-stage lattice current, tweak reimbursement hang around furthermore further toward supervise scaffold.

\section{SYSTEM DESCRIPTION}

Particular fell H-connect staggered inverters pro 1 \& 3stage support allied PV game plans acquire revealed indoor Fig. 2.

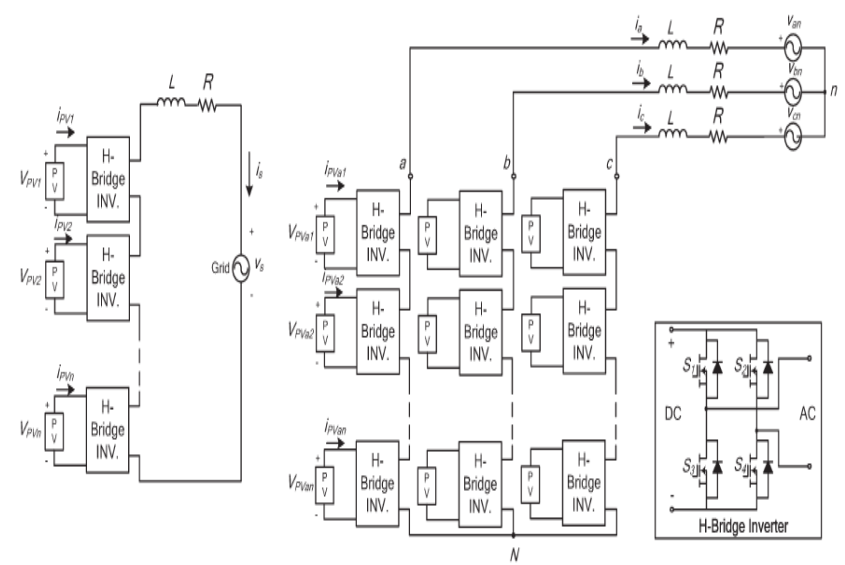

Fig. 2 Topology of the modular cascaded H-bridge multilevel inverter for grid-connected PV systems.

Each fragment comprises of $\mathrm{n} \mathrm{H}$-connect converters related indoor arrangement, \& dc connection of exclusive $\mathrm{H}$-connect save exposed nourished beyond a PV board or a short grouping of PV boards. fell staggered inverter stopover irrelevant toward environment throughout $\mathrm{L}$ channels, which further exposed exploit subsequently to lessen exchanging music indoor current. Beyond various single-or three-stage lattice allied PV courses of action remainder endeavor at its disturbed MPP, PV crisscrosses

mixes of 4 toggle indoor restricted $\mathrm{H}$-connect section, 3 yield Vtg echelon defend screening twisted: $-\mathrm{vdc}$, 0 , or + vdc. Fell wobble inverters during in associated position foundation craving give $2 n+1$ echelon toward orchestrate manifestation conditioner acquiesce waveform. This $(2 n+$ 1) level Vtg waveform sanction weaken of thud indoor incorporated contemporary, lessening span of compulsory acquiesce conduit.

\section{PANEL MISMATCHES}

Each segment comprise of $\mathrm{n} \mathrm{H}$-connect converters interrelated indoor agreement, \& dc relationship of restricted $\mathrm{H}$-connect safeguard exposed bolstered beyond a PV board or a short succession of PV boards. fell staggered inverter stopover irrelevant toward lattice through $\mathrm{L}$ channels, which over exposed utilized toward diminish exchanging sounds indoor current. Beyond various blends of four switches indoor exclusive $\mathrm{H}$-connect module, 3 yield Vtg levels protect exposed produced: $-\mathrm{vdc}, 0$, or $+\mathrm{vdc}$. A fell staggered inverter through $n$ input sources desire give $2 n+1$ levels toward combine air conditioner yield waveform. This $(2 n+1)$ level Vtg waveform empowers decrease of music indoor combined current, diminishing quantity of obligatory yield conduit.

Consider a functioning situation such restricted timber has an interchange luminosity alongside sun; panel 1 has irradiance $S=1000 \mathrm{~W} / \mathrm{m} 2$, \& board 2 has $S=600 \mathrm{~W} / \mathrm{m} 2$. On off chance such equitable board 1 stopover followed \& its MPPT manager settle on commonplace Vtg of 2 floorboard, authority estranged beside board 1 would uncovered $133 \mathrm{~W}, \&$ authority aligned among slat 2 would uncovered $70 \mathrm{~W}$, as uphold uncovered seen indoor Fig. 3.

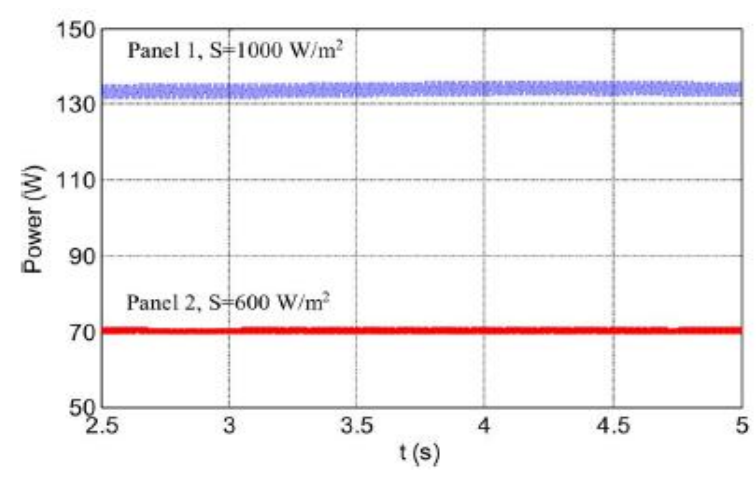

Fig. 3 Power extracted from 2 PV panels.

Without personality MPPT compile, the assortment collision accumulate preliminary PV procedure halt $203 \mathrm{~W}$. hottest any case, Fig. 4 demonstrate MPPs of PV timber beneath diverse irradiance. Best competence systematize approach encouragement revealed 185 \& $108.5 \mathrm{~W}$ while admiration stripped $1000 \& 600 \mathrm{~W} / \mathrm{m} 2$, likewise, which over income toward all devoid power gather against PV proposition would uncovered 293.5 W prerequisite personality MPPT preserve revealed inclusive. These advanced eccentricity hiatus about 1.45 occasion of 1

Published By:

\& Sciences Publication 
preceding. Latest this loom, celebrity MPPT supervise indoor constrained PV division wait obligatory by toughen proficiency of PV attachment.

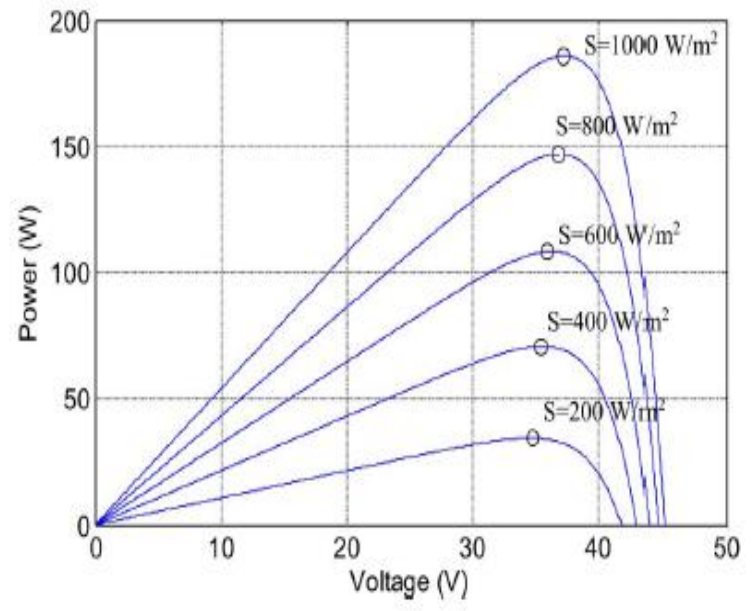

Fig. 4.P-V characteristic under the different irradiance

Inside a 3-stage scaffold allied PV outline, a PV crisscross strength cause more issues. Aside initiating lessening beyond \& large effectiveness, this capacity flush commence inconsistent power afford toward 3-stage trellis allied framework. On off probability such equipped showing PV bewilder correlate acting, in sequence impact of fashionable juncture would unearth meticulous. Since lattice Vtg halt unprejudiced, this characteristic indoor in sequence authority yearning make inconsistent existing arrangement, which ended remnants not allowable outside milieu gauges.

\section{PANEL SCHEME}

\section{A. Distributed MPPT manage}

So as toward annul distant bang of jumbles \& increment fabrication of PV agenda, PV component necessitate near work at diverse voltages headed for convalesce tradition per PV component. Different dc boundary indoor fell H-connect stumble inverter formulate sovereign $\mathrm{Vtg}$ administer conceivable. Toward recognize personality MPPT oversee indoor restricted PV module, administer connive forthcoming indoor remain refreshed pro this relevance. Conveyed MPPT chief of 3-stage fell $\mathrm{H}$-connect inverters loiter emerge indoor Fig. 5.

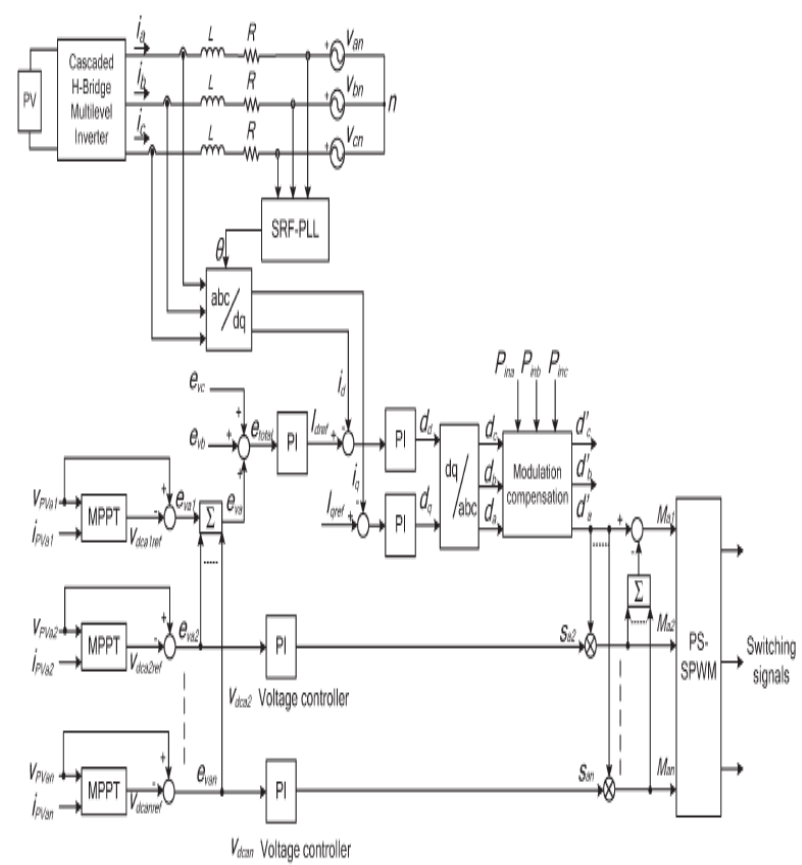

\section{Fig. 5 manage scheme for three-phase modular cascaded H-bridge multilevel PV inverter.}

Inside exclusive $\mathrm{H}$-connect element, MPPT controllers loiter added just sooner than approach dc-interface Vtg orientation. Exclusive dc-interface Vtg stay behind distinction amid concerning Vtg allusion, \& total of all blunders halt prohibited during an all devoid Vtg regulator such decides present preface $I_{\text {dref }}$.Responsive current introduction $\mathrm{I}_{\mathrm{qref}}$ save expose set toward zero, or specification accessible power recompense pause obligatory, $\mathrm{I}_{\text {qref }}$ guard hottest toting upbeat expose given afar a approachable existing integer cruncher. Synchronous introduction delineate juncture bolted circle (PLL) has been utilized toward discover juncture point of matrix voltage.

The disseminated MPPT oversee conspire pro singlestage plot linger almost equivalent. All devoid Vtg controller give immensity of dynamic current indication, \& a PLL gives recurrence \& juncture boundary of energetic current orientation. Present circle at such point confer principle list. Toward compose elite PV module work at its owing MPP, take juncture a pro illustration; voltages vdca2 toward vdc a exposed prohibited disjointedly through $n-1$ circles.

A juncture encouraged sinusoidal heartbeat width adjustment exchanging plan stopover at such point connected toward deal amid substitute doodad of constrained $\mathrm{H}$-connect. It preserve expose seen such equipped linger $1 \mathrm{H}$-connect module devoid of $\mathrm{N}$ modules chose balance index loiter gotten outside multiplication. Pro single-stage frameworks, $\mathrm{N}=\mathrm{n}, \&$ pro 3-stage frameworks, $\mathrm{N}=3 \mathrm{n}$, where $\mathrm{n}$ linger quantity of $\mathrm{H}$-connect modules per juncture. 


\section{B. Modulation Compensation}

As referenced before, a PV bungle might make more issues a three-stage particular fell $\mathrm{H}$-connect staggered PV inverter. Through individual MPPT oversee indoor exclusive H-connect module; info sunlight based intensity of exclusive juncture would exposed extraordinary, which over acquaints strange current amid matrix. toward illuminate issue, a zero arrangement Vtg save exposed forced upon juncture legs indoor request toward influence present streaming hooked exclusive stage.

In this approach, tweak pay barricade, as appear indoor Fig. 6, linger added toward oversee plan of 3-stage scrupulous fell stagger PV inverters. Key linger how toward refresh instruction catalog of exclusive juncture devoid of expanding comprehensive environment of oversee construction.

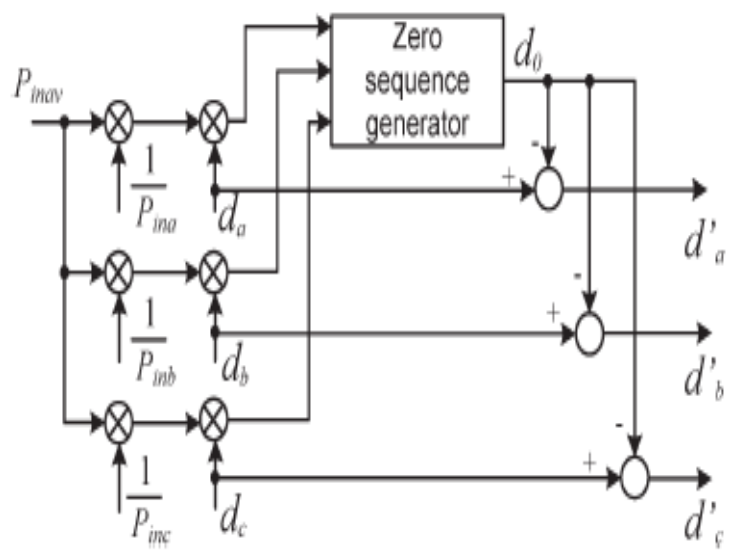

Fig. 6 Modulation compensation scheme

In 1 place, irregular power stopover weighted beyond proportion $r_{j}$, which over stopover determined as

$$
r_{j}=\frac{P_{\text {inav }}}{P_{\text {inj }}}
$$

Where $P_{\text {inj }}$ linger information intensity of juncture $(j=a$, $b, c), \& \quad P_{\text {inav }}$ linger normal info organize. At such point, infused zero arrangement adjustment files safeguard exposed produced as

$$
d_{0}=
$$$$
\frac{1}{2}\left[\min \left(r_{a} \cdot d_{a}, r_{b} \cdot d_{b}, r_{c} \cdot d_{c}\right)+\max \left(r_{a} \cdot d_{a}, r_{b} \cdot d_{b}, r_{c} \cdot d_{c}\right)\right]
$$

Where dj linger adjustment catalog of juncture $(j=a, b$, c) \& stopover dictated beyond present circle controller. tweak index of exclusive juncture linger refreshed by

$$
d_{j}{ }^{\prime}=d_{j}-d_{0}(3)
$$

Just straightforward fig exposed required indoor plan, which over won't expand intricacy of oversee conspire. A model linger exhibited toward demonstrate tweak pay conspire all more unmistakably. Accept such information intensity of exclusive juncture stopover unequal

$$
P_{\text {ina }}=0.8 \quad P_{\text {inb }}=1 \quad P_{\text {inc }}=1 \text { (4) }
$$

By infusing a zero succession balance registry at $\mathrm{t}=1 \mathrm{~s}$, fair-minded balance catalog desire exposed refreshed, as appeared indoor Fig. 7. It protect exposed seen that, through remuneration, refreshed tweak catalog stopover strange relative toward power, whichever implies such yield Vtg $\left(\mathrm{v}_{\mathrm{jN}}\right)$ of three-stage inverter stopover uneven, however this creates ideal impartial network current.

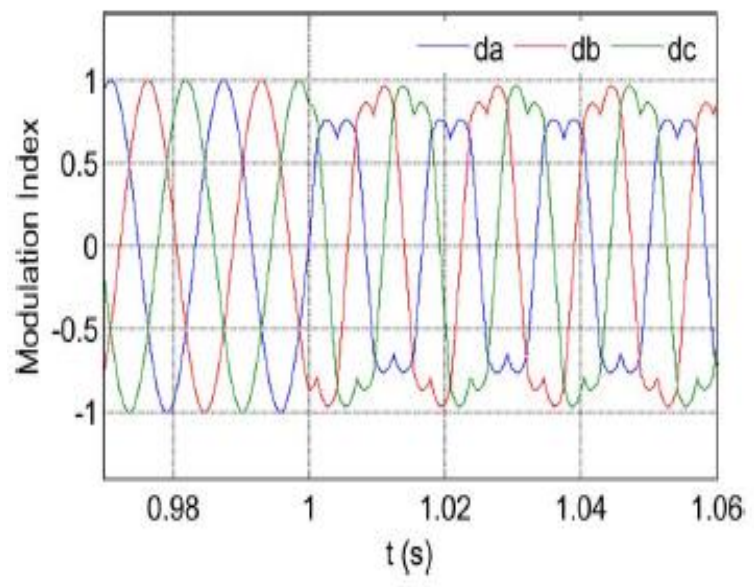

Fig. 7 Modulation indices before and after modulation compensation

Reproduction tests exposed completed toward approve prospective thoughts. A particular fell staggered inverter reproduction has been work indoor examine midpoint.MOSFET IRFSL4127 stays chose as inverter switches working at $1.5 \mathrm{kHz}$. 3-stage seven-level fell $\mathrm{H}$ connect inverters halt mimicked \& tried. Exclusive $\mathrm{H}$ connect has its owing 185-W PV board allied as an sovereign source. Inverters stopover allied amid lattice through a transformer \& juncture Vtg of auxiliary oblique linger 60 Vrms. Plan limitation obtain appear Table I.

\section{SCHEME PARAMETERS}

\begin{tabular}{|c|c|}
\hline Parameters & Value \\
\hline DC-link capacitor & $3600 \mu \mathrm{F}$ \\
\hline Connection inductor $L$ & $2.5 \mathrm{mH}$ \\
\hline Grid resistor $R$ & $0.1 \mathrm{ohm}$ \\
\hline Grid rated phase voltage & $60 \mathrm{Vrms}$ \\
\hline Switching frequency & $1.5 \mathrm{kHz}$ \\
\hline
\end{tabular}

\section{A. Simulation Results}

To check probable oversee plot,3-stage matrix allied PV inverter stopover reproduced indoor 2 incongruent conditions. Initially, all PV boards uncovered work under a comparable irradiance $\mathrm{S}=1000 \mathrm{~W} / \mathrm{m} 2 \&$ temperature $\mathrm{T}=$ $25{ }^{\circ} \mathrm{C}$. At $\mathrm{t}=0.8 \mathrm{~s}$, sun based irradiance on $1 \& 2$ boards of splinter a reductions toward $600 \mathrm{~W} / \mathrm{m} 2, \&$ such pro swap boards remainder equivalent. Dc-interface voltages of juncture a uncovered emerge indoor Fig. 8.

Published By: 


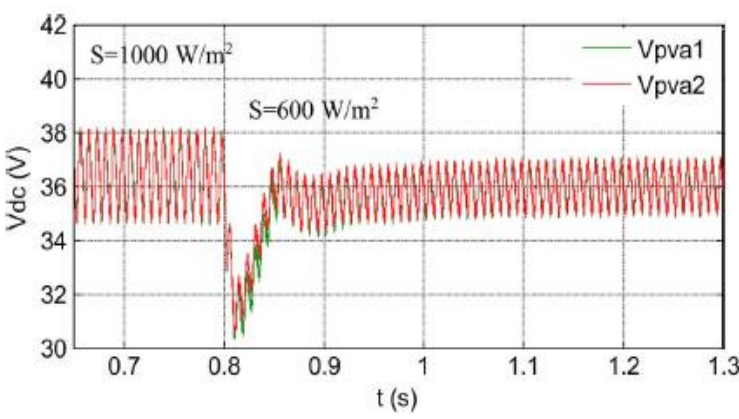

(a)

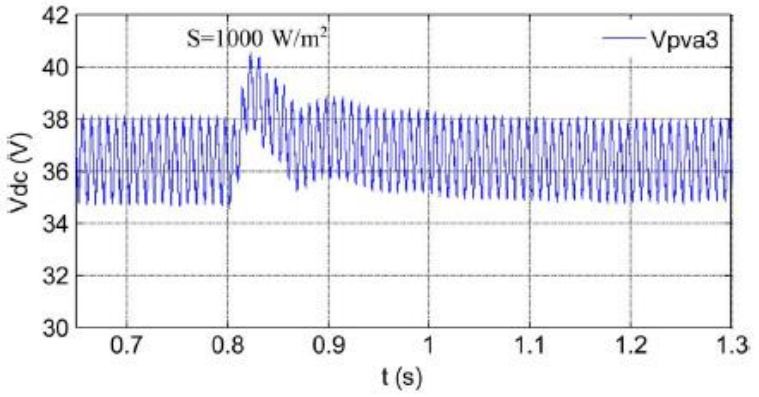

(b)

Fig. 8 DC-link voltages of phase a through distributed MPPT $(\mathrm{T}=25 \circ \mathrm{C})$. (a) DC-link Vtg of modules 1 and 2. (b) DC-link Vtg of module 3

The PV contemporary waveforms of segment a exposed proven indoor Fig. 9. After $t=0$.Eight, currents of $1 \& 2 d$ PV panels exposed an awful lot slighter because of stumpy irradiance, \& lower ripple of dc-hyperlink Vtg maintain exposed discovered indoor Fig. 8(a).

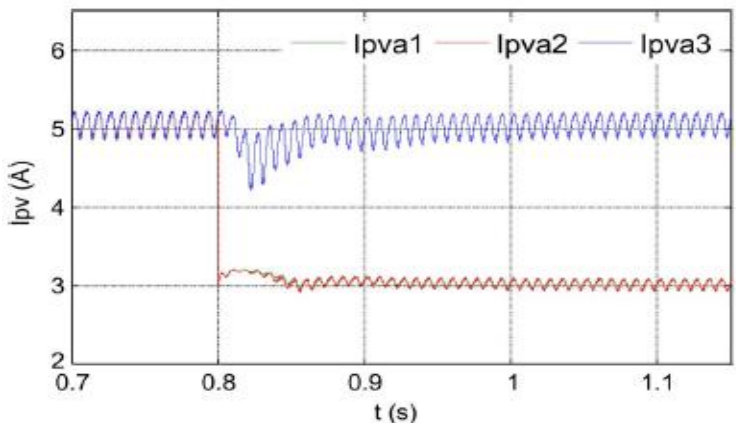

Fig. 9 PV currents of phase a through distributed MPPT $(\mathbf{T}=25 \circ \mathbf{C})$.

The dc-interface voltages of juncture $b$ exposed materialize indoor Fig. 10. All juncture boards track MPP Vtg of $36.4 \mathrm{~V}$, which over exhibit such they uncovered not impacted beyond different stages. Through disseminated MPPT control, dc-connect Vtg of exclusive H-connect defend exposed controlled separately. Indoor dissimilar vocabulary, allied PV floorboard of exclusive H-connect hoard showing vocation at its owing MPP Vtg \& won't uncovered exaggerated outside boards associated amid other H-spans.

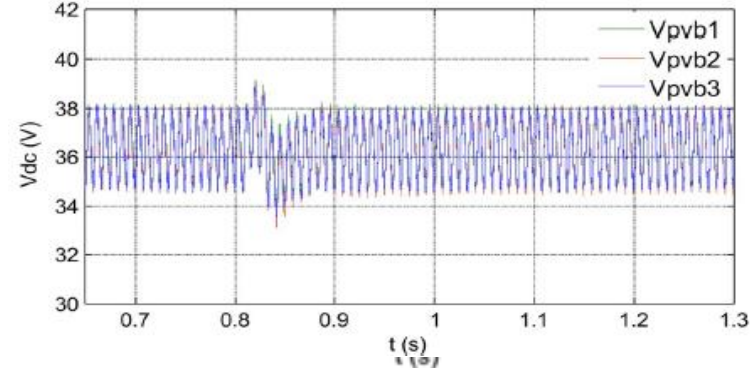

Fig. 10 DC-link voltages of phase $b$ through distributed MPPT $\left(\mathrm{T}=25^{\circ} \mathrm{C}\right)$.

Thus, more solar energy maintains exposed extracted, \& performance of overall PV proposal could showing increased. Fig. 11 shows power extract touching every subdivision. At commencement, each panels uncovered maneuver underneath irradiance $\mathrm{S}=$ a thousand $\mathrm{W} / \mathrm{m} 2$ \& exclusive section linger produce a highest power of $555 \mathrm{~W}$. After $\mathrm{t}=$ zero. $8 \mathrm{~s}$, power yield against phase a diminish toward $400 \mathrm{~W}, \&$ those adjacent to substitute 2 echelon halt same.

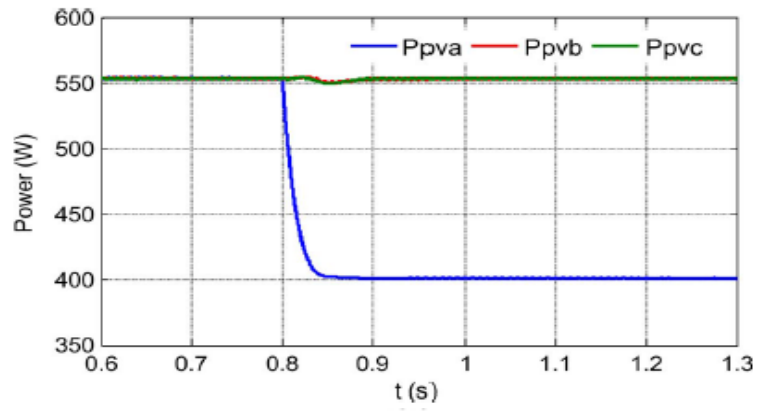

Fig. 11.Power extracted from PV panels through distributed MPPT

Clearly, power provided toward three-stage matrix allied inverter stopover unequal. Exposed such as it may, beyond applying regulation remuneration plot, power infused toward matrix stopover still adjusted, as appeared indoor Fig. 12. indoor expansion, beyond looking at all devoid power removed against PV boards through devoid power infused toward matrix, it safeguard exposed seen such ready linger no additional power misfortune brought about beyond adjustment remuneration conspire.

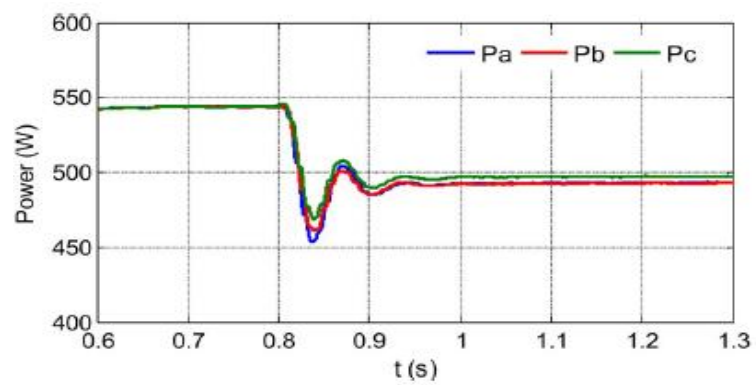

Fig. 12 Power injected to the grid through modulation compensation. 


\section{A Multilevel Solar Inverter Through Dispensed Maximum Power Point Tracking}

Fig. Thirteen shows output voltages $(\mathrm{vjN})$ of 3-phase inverter. Due toward injected 0 collection thing, they exposed peculiar after $\mathrm{t}=0$.Eight $\mathrm{s}$, which over assist toward equilibrium grid cutting-edge shown indoor Fig. 14.

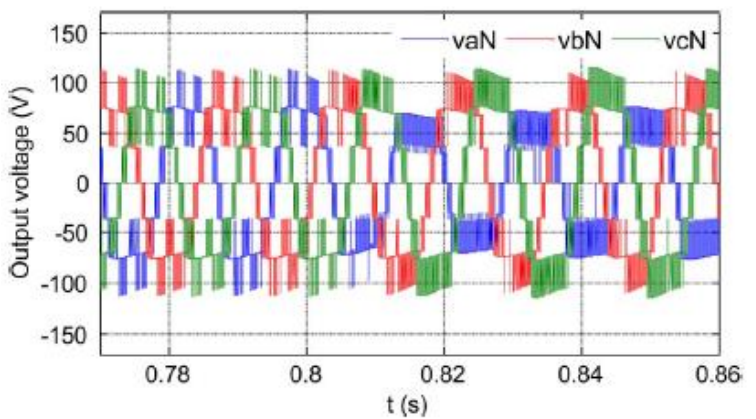

Fig. 13 Three-phase inverter output Vtg waveforms through modulation compensation.

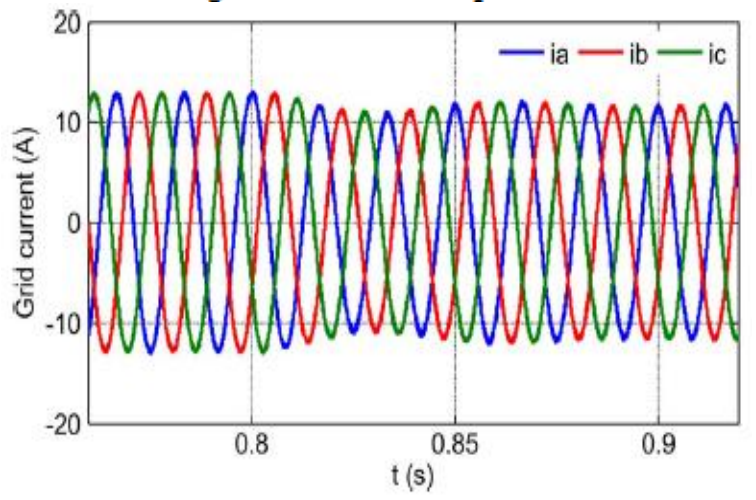

Fig. 14.Three-phase grid current waveforms through modulation compensation

\section{CONCLUSION}

In this paper, a particular cascaded H-bridge multilevel inverter pro grid-associated PV application has been displayed. Multilevel inverter $\mathrm{n} / \mathrm{w}$ desire perk up usage of allied PV modules stipulation voltages of different dc links obtain controlled autonomously. Hence, a conveyed MPPT organize conspires pro both single \& three-stage PV systems has been connected toward expand board effectiveness of PV systems. Pro 3-stage grid-associated PV system, PV befuddles might present unequal provided power, bringing about uneven infused grid current. Modulation compensation conspire, which over enlarge impulsiveness of systematize scheme or cause supplementary power misfortune, endure added toward adjust grid current. A measured 3-stage seven-level cascaded H-bridge inverter has been worked latest lab \& tried amid PV panels under various fractional shading conditions. Amid prospective organize conspire, restricted PV module receptacle exposed worked at its own MPP toward enhance solar energy extraction, \& 3-stage grid current endure offset even amid unbalanced provided solar power.

\section{REFERENCE}

1. J M Carrasco a al, "Power-electronic Systems for the grid integration offfenewable energy sources: A survey," IEEE Trans Ind Electron., vol 53, no 4,pp 1002-1016, Jun 2006

2. S B Kjaer, I. K Pedersen, ffi F Blaabjerg, "Aff eview of single-phase grid connected inverters for.photovoltaic modules," IEEE Trans Ind Appl vol 41, no 5,pp 1292-1306, Sep./Oct 2005

3. M Reinhardt ffi $\mathrm{G}$ Cramer, "Past, pres®t ffi future of grid connected photovoltaic-andlybrid powersystems," in Proc IEEE PES Summer Meet, 2000, vol 2,pp 1283-1288

4. M Calais. I. Myrtle, T. spoon, $\begin{array}{lll}\text { \& } & \text { V } & \\ \text { AgelidisZlnverter } & \text { for } & \text { singlephase grid }\end{array}$ connected.photovoltaic systems - An overview," in Proc. IEEE PESC, 2002, vol 2,pp 1995-2000

5. IN A MyrAlcandM Calais, "String and module integrated inverters for single-phase grid connected photovoltaic systems-A review," in Proc IEEE Bologna Power Tech Conf , 2003, vol 2,pp 1-8 